\title{
Der Weg entsteht im Gehen
}

\author{
K. Thieme \\ Institut für Geographie, Universität Augsburg, Augsburg, Germany \\ Correspondence to: K. Thieme (karin.thieme@geo.uni-augsburg.de)
}

Meyer, F.: Der Weg entsteht im Gehen, Eine qualitative Einzelfallstudie zur Raumwahrnehmung und Mobilität von Jugendlichen mit Down-Syndrom, mit zahlreichen farbigen und Schwarz-Weiß-Abbildungen, gebunden, Peter Lang AG Bern/CH et al., Bern, 231 S., ISBN-13: 978-3-0343-0521-1, 2010 .

Die vorliegende Studie von Franziska Meyer wurde im Rahmen eines Dissertationsprojekts an der Universität Bern angefertigt. Die Autorin studierte Heilpädagogik und Allgemeine Ökologie an den Schweizer Universitäten Freiburg und Bern. Dort erfolgte im Jahr 2009 die Promotion im Fach Sozialgeografie. Franziska Meyer arbeitete bereits als Pädagogin in der Kinder- und Jugendpsychiatrie in Bern. Sie war außerdem Co-Leiterin der Kriseninterventionsstation des Jugendamtes Bern und leitete u.a. interkulturelle Projekte. Heute ist Franziska Meyer wissenschaftliche Mitarbeiterin am Zentrum für universitäre Weiterbildung der Universität Bern sowie freie Mitarbeiterin des Marie-MeierhoferInstituts in Zürich und Dozentin an der Pädagogischen Hochschule Bern.

Die Beschäftigung mit dem Thema Handicap und Behinderung hat in der Sozialgeografie eine gewisse Tradition. Einen geografischen Niederschlag findet das Thema insbesondere mit der Erstellung z.B. von Stadtplänen für Menschen mit Behinderung. Franziska Meyer hat mit der vorliegenden Arbeit den Themenkreis erheblich erweitert. Dies zeigt sich bereits in der Gliederung ihrer Studie, die ein breites Spektrum sozialgeografischer Diskussion umfasst: Die Thematik ist mit wichtigen Forschungsfeldern der Sozialgeografie verknüpft wie z.B. der Annäherung an den Raumbegriff, die Themen Raumwahrnehmung und -aneignung, Orientierung im Raum sowie dem Thema Mobilität. Speziell auf die Thematik von Jugendlichen mit Down-Syndrom zugeschnitten, betritt Franziska Meyer wissenschaftliches Neuland.

Franziska Meyer stellt in ihrer Studie die betroffenen Jugendlichen als Akteure im öffentlichen Raum dar, indem sie sich dem öffentlichen Raum als Lern- und Bewegungsort für
Kinder und Jugendliche nähert. Auf der Grundlage qualitativer sozialgeografischer Forschung führt die Autorin Fallstudien durch, die sie mit Hilfe von visuellen Methoden empirisch begründet. Aufgrund eingeschränkter Sprachkompetenzen bei Jugendlichen mit Down-Syndrom setzt Franziska Meyer v.a. die Fotografie als partizipative Methode ein. Sie greift damit visuelle als auch ethnographische Methoden auf, die sie teilweise selbst weiter entwickelt.

Für Menschen mit Behinderung und damit auch Jugendliche mit Down-Syndrom ist es nicht selbstverständlich, ohne Begleitung zu Fuß zu gehen, den Bus zu nehmen, alleine und ohne fremde Hilfe den Weg zu finden. Behinderung darf jedoch nicht ausgrenzen, v.a. wenn dies den öffentlichen Raum mit seinen unterschiedlichsten Funktionen betrifft. Beim Thema Behinderung geht es nicht um Einschränkung, sondern um Teilhabe, Integration und Kompetenz. Dies bedeutet im Rückschluss, dass Menschen mit Behinderung lernen müssen, sich zurecht zu finden und ebenso mobil zu sein wie andere Verkehrsteilnehmer, und damit ihre ganz persönliche Freiheit und Selbständigkeit zu haben.

Das Down-Syndrom beim Menschen basiert auf einer Genmutation, von der das 21. Chromosom betroffen ist (Trisomie 21). Das Down-Syndrom ist damit keine Krankheit, sondern eine - wenn auch nicht abänderbare - genetische Besonderheit. Neben veränderten körperlichen Fähigkeiten wie beispielsweise gestörte Motorik oder Sensorik weisen Menschen mit Down-Syndrom auch eine Beeinträchtigung ihrer kognitiven Fähigkeiten sowie Defizite der sozialen Kompetenz auf. Mit einer von 700 Geburten ist das Down-Syndrom die häufigste angeborene Chromosomenveränderung.

Typisch für Kinder mit Down-Syndrom und damit für die Fähigkeit zu räumlicher Wahrnehmung ist die Entwicklung kognitiver und motorischer Fähigkeiten. Kinder mit Down-Syndrom benötigen mehr Zeit, auf Reize zu reagieren, was für das Verhalten im (öffentlichen) Raum ein wichtiger Aspekt ist. Hinzu kommen oftmals Seh- und Hörstörungen, die ebenfalls die Orientierung im öffentlichen Raum erschweren. Mobilität und das Sich-Bewegen im Raum ist ein wichtiger Baustein zur Erreichung des von Vereinen, 
Verbänden, Eltern und natürlich den Betroffenen mit DownSyndrom selbst angestrebten Ziels einer vollständigen gesellschaftlichen Integration und vorbehaltlosen Akzeptanz im Alltag.

Mobilität bzw. mobil zu sein gehört zu den Grundbedürfnissen des Menschen. Menschen mit diversen Handicaps sind oftmals in ihrer Mobilität eingeschränkt. Ihre Geografien sind quasi behindert, in der Regel bedingt durch Hindernisse im täglichen Umgang mit Raum. Franziska Meyer legt ihrer Studie einen physisch-räumlichen Mobilitätsbegriff zugrunde. Zwischen der Art der Fortbewegung bzw. der Bewegung im öffentlichen Raum und der sozialen Positionierung eines Menschen besteht ein enger Zusammenhang. Umgekehrt hat die Position im sozialen Raum Auswirkungen auf die Mobilitätspraxis des Menschen, sowie den Lebensstil und die jeweilige Lebenssituation. Mobil zu sein bedeutet Loslösung von Anderen, sich individuell und unabhängig zu bewegen, Räume selbst zu erkunden. Inwieweit dies bei Jugendlichen mit Down-Syndrom der Fall ist, erörtert Franziska Meyer in ihrer Studie.

Der öffentliche Raum ist im Grunde allen Menschen vorbehalten, über seine Nutzung entscheiden jedoch nicht die Nutzer und Nutzerinnen und damit Privatpersonen. Der öffentliche Raum ist Spiegel der Gesellschaft. Unser Verständnis von Öffentlichkeit bedingt unser Grundverständnis von öffentlichem Raum: er ist Raum für Kommunikation und Austausch, Raum des Widerspruchs aber auch des Anspruchs. Im Speziellen stellt der öffentliche Raum für Kinder und Jugendliche einen Ort des Lernens, der Sozialisierung und der Bewegung dar. Kinder mit einer geistigen Behinderung unterscheiden sich von Kindern ihrer Altersklasse durch gewisse Defizite intellektueller Fähigkeiten. Sie tun sich schwer, logische Zusammenhänge zu erfassen und nachzuvollziehen. Dies führt dazu, dass sie aus dem öffentlichen und damit Gesellschaftsraum oftmals ausgegrenzt werden. Die „Inseln“, die sie sich dann logischerweise aneignen, sind nicht nur qualitativ beschränkt, sondern auch spezialisiert. Mit Hilfe von Individualität und Selbstbild schaffen sie sich eine Sonderwelt.

Franziska Meyers Arbeit basiert auf Einzelfallstudien. Die ProbandInnen waren zwischen 15 und 18 Jahre alt. Voraussetzungen waren insbesondere, dass sie sich frei im Raum bewegen und eigene Wege finden konnten, aber auch Interesse und Lust an dem Projekt hatten. Das freie räumliche Bewegen kristallisierte sich jedoch als größte Hürde für das Projekt heraus, d.h. die ProbandInnen waren in ihrem autonomen Bewegungsspielraum teils stark eingeschränkt. Ein besonderes Problem war außerdem, dass es zur Erfassung des räumlichen Wissens und der Orientierungsfähigkeit bei Kindern/Jugendlichen bislang keine ausgewiesene Methode gibt und diese erst iterativ entwickelt werden musste. Die Datenerhebung erfolgte von Februar 2007 bis Juni 2008. Die gewonnenen Daten wurden verschiedenen Auswertungsmöglichkeiten unterzogen. Der Untersuchung lag ,aufgrund der theoretischen und methodologischen Ungewissheit ein abduktives Vorgehen zugrunde" (S. 69).

Das gewonnene Datenmaterial umfasste mittels GPS erstellte, kommentierte geografische Karten, Mental Maps, Feldbegehungen sowie die Fotodokumentation neu zu erlernender Routen. Damit konnten die Aktionsradien erkannt und zugeordnet werden. Durch Interviews wurden die Ergebnisse vertieft; denn allein aufgrund der GPS-Auswertung ist es beispielsweise nicht möglich zu sagen, ob sich die Aktionsradien aufgrund eingeschränkter körperlicher Mobilität der ProbandInnen ergeben oder möglicherweise aus der Angst der Eltern resultieren. Mit Hilfe der Mental Maps wurden die Ergebnisse konkretisiert; eine Vorgehensweise, die bislang in der sozialgeografischen Forschung bei Menschen mit Behinderung noch nicht erprobt wurde. Die abstraktminimalistischen Kartogramme geben Hinweise auf Landmarken und Streckenverläufe, z.B. wichtige Haltestellen des ÖV. Dennoch kommt Franziska Meyer zu dem Ergebnis, dass diese Methodik an ihre Grenzen stößt und nicht als Mittel für Jugendliche mit Down-Syndrom geeignet ist, ihre räumliche Umgebung darzustellen. Deshalb führte die Autorin mit den ProbandInnen Ortsbegehungen durch, um die Mental Maps zu konkretisieren. Dabei traten ähnliche Probleme zu Tage wie bei den Interviews: die knapp gehaltenen Kommentare der ProbandInnen führten zu keinen befriedigenden Ergebnissen, bewiesen jedoch, dass sie sich im öffentlichen Raum orientieren und räumliche Qualitäten aufzeigen konnten. Franziska Meyer führt deshalb in einer zweiten Methodenrunde die Fotografie als partizipatives Hilfsmittel ein. Die Jugendlichen hatten keine Probleme, z.B. ihre Schulwege umfangreich zu dokumentieren. Sie verfügten durchaus über ein Konzept und Wissen der Wege bzw. Routen und konnten diese anhand der Fotos rekonstruieren. Franziska Meyer folgert daraus, dass die ProbandInnen durchaus über besondere visuelle Wahrnehmungsfähigkeiten verfügen. Sie ließ somit in einem weiteren empirischen Schritt die ProbandInnen mit Hilfe von Fotografien neue Wege erschließen. Diese Analyse der fotografischen Produkte erbrachte schließlich wertvolle Hinweise für die Auswahl der Landmarken und Orientierungshilfen. Die Erkenntnisse wurden mittels Feldbegehungen überprüft und validiert; ,,denn nur wenn [die ProbandInnen] in der Lage sind, anhand ihrer selbst gewählten Hinweise und Landmarken den neuen Weg zu gehen, kann mit Gewissheit davon ausgegangen werden, dass es sich dabei um Orientierungshilfen handelt, die ihnen dazu dienen, sich im öffentlichen Raum zu bewegen" (S. 171). Zum Abschluss der Forschungsarbeiten zeigten die ProbandInnen, dass sie mit Hilfe personalisierter visueller Reiseführer auf der Grundlage von Fotografien in der Lage waren, auch neue Wege erfolgreich zu bewältigen.

Franziska Meyer stellt nach gründlicher Aus- und Bewertung der Empirie und Methodik fest, dass sich die Strategien von Jugendlichen mit Down-Syndrom, sich Wege zu erschließen, nur unwesentlich von nichtbehinderten NutzerInnen des öffentlichen Raums unterscheiden. Eigene Erfahrung 
und subjektive Bedeutungsgebungen sind wichtige Orientierungshilfen für Routenwissen. Ihre egozentrische Perspektive erlaubt es ihnen jedoch nicht, gleichzeitig ein Überblickswissen über den Raum zu gewinnen. Jugendliche mit DownSyndrom sind aber grundsätzlich in der Lage, ,sich dieses Überblickswissen durch eigentätige Exploration und die Erweiterung ihres Erfahrungsspielraums anzueignen“" (S. 202).

Für Kinder und Jugendliche sind öffentliche Räume Lebensräume, aber oftmals auch Orte der Restriktion und Barrieren. Dies trifft auf Jugendliche mit Down-Syndrom fast sprichwörtlich zu. Öffentlichen Raum autonom zu nutzen, hängt stark von der Fähigkeit zur geografischen Orientierung und eigenständiger Mobilität ab. Gestützt auf sozialgeografische und (heil)pädagogische Grundlagen liefert Franziska Meyers Studie konkrete Antworten auf die Frage der Raumaneignung und Orientierung von Jugendlichen mit DownSyndrom. Dabei weist die Autorin nach, dass Jugendliche mit Down-Syndrom den öffentlichen Raum bewusst und auch differenziert wahrnehmen. Sie orientieren sich mit Hilfe selbst gestalteter Feldbücher und eignen sich den öffentlichen Raum partiell an. Dies führt zu einer Erhöhung ihrer Mobilität. Als ExpertInnen in eigener Sache bzw. ihrer Lebenswelt demonstrieren sie ihre Wahrnehmung öffentlichen Raums und wie sie sich darin orientieren. Die Studie stellt einen methodisch wie inhaltlich innovativen Beitrag zur kompetenzorientierten Forschung mit Kindern und Jugendlichen mit Down-Syndrom dar. Franziska Meyer betritt oftmals Neuland und gewinnt damit mutige und hochinteressante Ergebnisse. Sie kommt zu dem vielleicht überraschenden Ergebnis, dass sich ,Jugendliche mit Down-Syndrom bezüglich der Strategien, sich Wege zu erschließen und im öffentlichen Raum zu orientieren, kaum von nichtbehinderten Benutzer/innen ... unterscheiden“" (S. 212). Obwohl es in den speziell untersuchten Fällen nicht unbedingt möglich ist, diese zu verallgemeinern, ist es dennoch möglich, Jugendliche mit Down-Syndrom als Experten zu respektieren und aus den Ergebnissen der Studie interessante Kriterien zur Gestaltung öffentlicher Räume abzuleiten; zumal vor dem Hintergrund einer alternden Gesellschaft auch Handicaps künftig zunehmen werden und viele Disziplinen, die sich mit „Raum“ auseinandersetzen, sich auch auf die neuen Verhältnisse und Voraussetzungen einstellen müssen.
Der ARBEITSKREIS DOWN-SYNDROM e.V. hat einen treffenden Slogan auf seiner Webseite formuliert, den man auch als Motto über Franziska Meyers Studie und deren richtungsweise Ergebnisse stellen könnte: „Damit Menschen mit Down-Syndrom da sein können, wo alle sind!“ 